\title{
New three-dimensional positioning algorithm through integrating TDOA and Newton's method
}

\author{
Junsuo $\mathrm{Qu}^{1 *}{ }^{*}$, Haonan Shi ${ }^{2 \dagger}$, Ning Qiao ${ }^{2}$, Chen $\mathrm{Wu}^{2}$, Chang Su${ }^{2}$ and Abolfazl Razi ${ }^{3}$
}

\begin{abstract}
Passive time difference location is an important method for passive location. There are fuzzy positioning, no solution, and low positioning accuracy with the spherical coordinate conversion method in the four-station TDOA positioning algorithm. Focusing on these problems, we proposed a combination of TDOA and iterative Newton's method. The positioning method uses the result obtained by the four-station TDOA location algorithm as the initial value of the iterative Newton's method and solves the problem of no solution and fuzzy positioning caused by the four-station TDOA location algorithm by using the spherical coordinates conversion method. By simulating the target at a height of $5 \mathrm{~km}$ and traveling at a constant speed for $40 \mathrm{~km}$, the positioning accuracy of the root mean square error is less than $45 \mathrm{~m}$, which can achieve the same positioning accuracy of TDOA based on the least square algorithm. As the baseline length increases, the positioning accuracy is better than the least square algorithm.
\end{abstract}

Keywords: TDOA, Newton's method, Fuzzy positioning, Insoluble position

\section{Introduction}

Moving target positioning plays a very important role in military and civil applications. The research about related technology is considered as a hot topic in many fields. Time difference of arrival (TDOA) is a method of positioning by time difference, since the distance of the signal source can be determined by measuring the time it takes for the signal to arrive at the monitoring station [1]. The accurate location of the source can be determined by finding its distance from three or more monitoring stations [2]. It features high accuracy, strong anti-interference capability, and fast positioning. Thus, it is widely used in passive localization [3]. TDOA is the location of the radiation source by processing the measurement data collected by three or more stations. Three-dimensional positioning requires four stations to generate three pairs of hyperboloids, the surface intersection lines and the intersection

*Correspondence: qujunsuo@xupt.edu.cn

† Junsuo Qu and Haonan Shi contributed equally to this work.

'School of Automation, Xi'an University of Posts and Telecommunications,

710121 Xi'an, China

Full list of author information is available at the end of the article points to determine the location of the radiation source. However, it may not result in a unique solution. Fuzzy regions may be obtained due to uncertainty of regions [4], or the intersection may be null due to measurement noise and error [5].

Fuzzy positioning affects the positioning accuracy [6]. The traditional methods proposed for eliminating fuzzy positioning and addressing problems with no solutions can be divided into two main categories. One is to eliminate fuzzy positioning by adding auxiliary measurement devices. The second is solving problems with no solutions by assuming a fixed target location. The studies in [7] and [8] proposed the use of high-precision measuring instruments by combining two algorithms based on least squares and iterative Newton's method. The study in [9] proposed that the number of the base stations participating in the measurement calculation are the master stations in turn. This method was raised in earlier literature and has been further developed. Another method is to make information or parameters more effective without adding auxiliary information. The spherical coordinate's system 
conversion belongs to the second category. Using the conversion from Cartesian coordinates to spherical coordinates, we can obtain a function of the distance difference between the target and the main station as well as that of the main station to the other three stations. By substituting this function into the equation used for coordinate conversion, we can evaluate the coordinates of the target. The hybrid method combining TDOA and spherical coordinate's conversion performs better in terms of positioning accuracy than using spherical coordinate's conversion only. The angles between the auxiliary stations are used when solving the distance difference function. When the distance is long, a tiny angle difference can affect positioning accuracy. The simulation in [10] demonstrates the positioning accuracy based on the spherical coordinate conversion method when the target is far from the base stations. However, it does not include simulating the accuracy when positioning low-altitude targets and does not consider the effect of each auxiliary station's angle. Furthermore, it does not combine the parametric approach with the spherical coordinate conversion.

In view of the shortcomings of these methods, we propose a joint algorithm, which is based on a four-station TDOA positioning system and uses spherical coordinate transformation to eliminate positioning ambiguity and non-solution phenomena. The positioning result is then used as the initial value of the Newton iteration, and finally, a hybrid algorithm of iterative operations is performed. The feasibility and reliability of the method are proved by simulation and data analysis.

\section{The analysis of fuzzy positioning and no solution}

A passive TDOA location system processes the data from radiation source collected by measurement stations and estimates the time difference of arrival. In threedimensional space, the time difference between two stations determines a hyperboloid with a pair of observatories as their focal points. The target needs at least three pairs of independent hyperboloid whose intersection defines the position, as such at least four base stations are required for positioning. For instance, the methods in [11] and [12, 13] used five and four base stations, respectively.

The TDOA positioning system consists of four base stations, we assume that the coordinate of the primary station is $\left(x_{0}, y_{0}, z_{0}\right)$, and the coordinates of each secondary station is $\left(x_{i}, y_{i}, z_{i}\right)_{i=1,2,3}$. As for four-station time difference positioning, assume that the spatial coordinate of the target is $(x, y, z)$, and the distance between the target and the primary station is $r_{0}$, the distance between the target and each secondary station is $r_{i}(i=1,2,3)$, and $\Delta r_{i}$ is the distance from the target to the primary station and the secondary stations, $c$ is the light propagation rate, and
$\Delta t_{i}(i=1,2,3)$ is the time difference from the target to the primary station and the secondary stations. It follows that

$$
\left\{\begin{array}{l}
r_{0}=\sqrt{\left(x-x_{0}\right)^{2}+\left(y-y_{0}\right)^{2}+\left(z-z_{0}\right)^{2}} \\
r_{i}=\sqrt{\left(x-x_{i}\right)^{2}+\left(y-y_{i}\right)^{2}+\left(z-z_{i}\right)^{2}} \\
\Delta r_{i}=r_{i}-r_{0}=c \cdot \Delta t_{i}
\end{array}\right.
$$

The above equations can be rewritten as [8]

$$
\left(x_{0}-x_{i}\right) x+\left(y_{0}-y_{i}\right) y+\left(z_{0}-z_{i}\right) z=k_{i}+r_{0} \cdot \Delta r_{i}
$$

where

$$
k_{i}=\frac{1}{2}\left[\Delta r_{i}^{2}+\left(x_{0}^{2}+y_{0}^{2}+z_{0}^{2}\right)-\left(x_{i}^{2}+y_{i}^{2}+z_{i}^{2}\right)\right]
$$

Equation (2) can be re-written in matrix form as follows:

$$
A X=F
$$

where

$$
\begin{aligned}
A & =\left[\begin{array}{lll}
x_{0}-x_{1} & y_{0}-y_{1} & z_{0}-z_{1} \\
x_{0}-x_{2} & y_{0}-y_{2} & z_{0}-z_{2} \\
x_{0}-x_{3} & y_{0}-y_{3} & z_{0}-z_{3}
\end{array}\right] \\
X & =[x, y, z]^{T} \\
F & =\left[\begin{array}{l}
k_{1}+r_{0} \cdot \Delta r_{1} \\
k_{2}+r_{0} \cdot \Delta r_{2} \\
k_{3}+r_{0} \cdot \Delta r_{3}
\end{array}\right]
\end{aligned}
$$

This TDOA-based localization system has three equations, but four unknowns $x, y, z, r_{0}$. Suppose that $r_{0}$ is one known, when $\operatorname{rank}(A)=3$, the estimated value of the target position will be

$$
\hat{X}=A^{-1} \cdot F
$$

Set

$$
A^{-1}=\left[\begin{array}{lll}
a_{11} & a_{12} & a_{13} \\
a_{21} & a_{22} & a_{23} \\
a_{31} & a_{32} & a_{33}
\end{array}\right]=\left[a_{i j}\right]_{3 \times 3}
$$

and the estimated position of the target would be

$$
\left\{\begin{array}{l}
\hat{x}=m_{1}+n_{1} \cdot r_{0} \\
\hat{y}=m_{2}+n_{2} \cdot r_{0} \\
\hat{z}=m_{3}+n_{3} \cdot r_{0}
\end{array}\right.
$$

where

$$
\left\{\begin{array}{l}
m_{i}=\sum_{j=1}^{3} a_{i j} \cdot k_{j} \\
n_{i}=\sum_{j=1}^{3} a_{i j} \cdot \Delta r_{i}
\end{array}\right.
$$

Substitute (10) into (2), a quadratic equation where $r_{0}$ represents an unknown can be obtained using

$$
a \cdot r_{0}^{2}+2 b \cdot r_{0}+c=0
$$

where

$$
\left\{\begin{array}{l}
a=n_{1}^{2}+n_{2}^{2}+n_{3}^{2}-1 \\
b=\left(m_{1}-x_{0}\right) n_{1}+\left(m_{2}-y_{0}\right) n_{2}+\left(m_{3}-z_{0}\right) n_{3} \\
c=\left(m_{1}-x_{0}\right)^{2}+\left(m_{2}-y_{0}\right)^{2}+\left(m_{3}-z_{0}\right)^{2}
\end{array}\right.
$$


The three-dimensional coordinates of the target can be obtained by solving (12). When $\Delta=b^{2}-a c=0$, Eq. (12) has a unique solution. When $\Delta=b^{2}-a c>0$ or $\Delta=b^{2}-a c<0$, Eq. (12) yields respectively a fuzzy position or no solution. To eliminate fuzzy positioning, other measurement information or prior information from the radiation sources is needed. Typically, no method solves the equations by assuming that the target height is known [14]]. In this paper, both cases of fuzzy positioning and no solution are addressed by using the spherical coordinate conversion.

\section{Using spherical coordinate conversion to eliminate fuzziness and no solution}

Figure 1 demonstrates conversion between the spherical and Cartesian coordinate systems. In Fig. $1, M$ is the aerial target; $O$ is the main station; and $A, B$, and $C$ are the three auxiliary base stations. $\theta$ is the angle between station $B$ and the $x$-axis, and $\alpha$ and $\beta$ denote the pitch and azimuth angles, respectively. Assume that $\varphi_{1}$ and $\varphi_{2}$ are the angles between $A, B$, and $C$, respectively. The length between the main station $O$ and $A, B$, and $C$ is denoted respectively by $L_{1}, L_{2}$, and $L_{3}$. In this figure, $O M$ and $M B$, respectively, $r_{0}$ and $\Delta t_{2}$ in Equi $(\mathrm{X}-\mathrm{Y})$. According to the spherical coordinate interpolation method for $\triangle M O B$, we have

$$
\left(r_{0}-\Delta r_{2}\right)^{2}=r_{0}^{2}+L_{2}^{2}-2 r_{0} L_{2} \cos (\beta-\theta) \cos \alpha
$$

After simplification, it converts to

$$
r_{0} L_{2} \cos (\beta-\theta) \cos \alpha-r_{0} \Delta r_{2}=\frac{1}{2}\left(L_{2}^{2}-\Delta r_{2}^{2}\right)
$$

Similarly, applying the same equation to $\triangle M O A$ and $\triangle M O C$, it follows that

$$
\left\{\begin{array}{l}
r_{0} L_{3} \cos \left(\beta-\theta-\varphi_{2}\right) \cos \alpha-r_{0} \Delta r_{3}=\frac{1}{2}\left(L_{3}^{2}-\Delta r_{3}^{2}\right) \\
r_{0} L_{2} \cos (\beta-\theta) \cos \alpha-r_{0} \Delta r_{2}=\frac{1}{2}\left(L_{2}^{2}-\Delta r_{2}^{2}\right) \\
r_{0} L_{1} \cos \left(\beta-\theta-\varphi_{1}\right) \cos \alpha-r_{0} \Delta r_{1}=\frac{1}{2}\left(L_{1}^{2}-\Delta r_{1}^{2}\right)
\end{array}\right.
$$

If we set

$$
L_{1}=L_{2}=L_{3}=L, k_{i}=\frac{1}{2}\left(L_{i}^{2}-\Delta r_{i}^{2}\right), i=1,2,3
$$

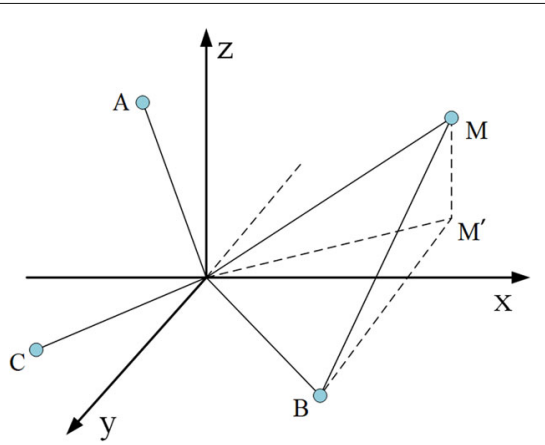

Fig. 1 Spherical coordinates and Cartesian coordinate systems then we obtain

$$
r_{0}=\frac{k_{2} \sin \varphi_{2}-k_{3} \sin \varphi_{1}-k_{1} \sin \left(\varphi_{2}-\varphi_{1}\right)}{\Delta r_{1} \sin \left(\varphi_{2}-\varphi_{1}\right)-\Delta r_{2} \sin \varphi_{2}+\Delta r_{3} \sin \varphi_{1}}
$$

\subsection{A Eliminating fuzzy positioning}

Addressing the fuzzy positioning and no-solution cases for four-station TDOA positioning system has been a major concern. Fuzzy positioning is due to obtaining multiple values for underdetermined positioning equations when the rank of $\mathrm{A}$ is below 3 , and the no-solution case is for over determined and inconsistent equations when the rank of A is above 3. Using spherical coordinate conversion, fuzzy positioning can be eliminated by integrating (18) and four-station TDOA location equations, as follows

$$
\left\{\begin{array}{l}
r_{i}=\sqrt{\left(x-x_{i}\right)^{2}+\left(y-y_{i}\right)^{2}+\left(z-z_{i}\right)^{2}} \\
\Delta r_{i}=r_{i}-r_{0}=c \cdot \Delta t_{i} \\
r_{0}=\frac{k_{2} \sin \phi_{2}-k_{3} \sin \phi_{1}-k_{1} \sin \left(\phi_{2}-\phi_{1}\right)}{\Delta r_{1} \sin \left(\phi_{2}-\phi_{1}\right)-\Delta r_{2} \sin \phi_{2}+\Delta r_{3} \sin \phi_{1}}
\end{array}\right.
$$

By solving this equation set, fuzzy positioning can be eliminated and the target value can be obtained with no ambiguity.

\subsection{B Eliminating the no-solution case}

After knowing the value of $r_{0}$, the distance between the target and the main base station, we can use conversion between the spherical and Cartesian coordinates to obtain

$$
\left\{\begin{array}{l}
x^{\prime}=r_{0} \cos \varphi \cos \alpha \\
y^{\prime}=r_{0} \sin \varphi \cos \alpha
\end{array}\right.
$$

and by the rotation of coordinates, we locate the target as

$$
\left\{\begin{array}{l}
x=x^{\prime} \cos \theta-y^{\prime} \sin \theta \\
y=x^{\prime} \sin \theta+y^{\prime} \cos \theta
\end{array}\right.
$$

It is easy to obtain the value of $z$ by equation

$$
z=\sqrt{r_{0}^{2}-x^{2}-y^{2}}
$$

The no-solution case is due to the inconsistency of the equation set itself. It can be eliminated by substituting (4) into (20), (21), and (22) top obtain the estimated target value.

From the above process, we know that if the time difference between the target to the master station and to the secondary stations is known, we can obtain the relation between time difference and $r_{0}$ and then calculate the three-dimensional coordinates of the target directly using the spherical coordinates conversion method [15]. To tackle different base station layouts, we only need to adjust different baseline lengths and angles between the base stations. 


\section{Spherical coordinate conversion and layout of stations}

TDOA localization and the spherical coordinate's conversion allow for adjusting the angles and baseline lengths between the base stations. However, the layout mode will also change correspondingly which impacts the positioning accuracy $[16,17]$.

Suppose that the time error of each base station is $10 \mathrm{~ns}$, each baseline length is $50 \mathrm{~km}$, and the site measurement error is $2 \mathrm{~m}$, correlation coefficient of the time measurement error and the flattened measurement error is 0.3 , and the positioning height is $10 \mathrm{~km}$. In this positioning system, we set the main station as the original point. We can simulate the positioning accuracy in star and triangular layouts. Simulation results are shown in Fig. 2.

From Fig. 2, the positioning accuracy of the star layout outperforms that of the triangular layout by $30 \%$. With the star layout, the closer the target and main station, the higher the location accuracy, hence the motion path will be circle alike. On the other hand, with triangular layout, if the positioning accuracy distribution is stronger, the target will be closer to the $x$-axis and the positioning accuracy will be higher. Therefore, when we use spherical coordinate system, the star layout can play a better role in positioning accuracy [18]. Although changing the angle between the base stations can enable us to locate the target, positioning accuracy will be far better when they are arranged in a star way.

\section{Newton's method}

After solving the fuzzy positioning and the no-solution cases and analyzing how positioning accuracy is affected by different station layouts, we can consider the estimated value as the initial value and use the Newton's iterative algorithm to enhance the results. Newton's method is an algorithm that uses gradient of a function similar to the gradient descent algorithm to iteratively solve optimization problems. It has been proven to converge faster than the gradient descent for many cases [19]. We start with an initial value and then start the iterative estimation until we get a solution satisfying certain estimation criteria. The closer the initial value to the true value, the better the convergence of this algorithm [20]. Therefore, it is significant to select an appropriate initial value to ensure an acceptable convergence rate. According to the conditions of Newton's algorithm, the established mode of target positioning system is converted into

$$
\begin{gathered}
f_{i}(x, y, z)=\sqrt{\left(x-x_{i}\right)^{2}+\left(y-y_{i}\right)^{2}+\left(z-z_{i}\right)^{2}}- \\
\sqrt{\left(x-x_{1}\right)^{2}+\left(y-y_{1}\right)^{2}+\left(z-z_{1}\right)^{2}}-c \Delta t_{i}
\end{gathered}
$$

and its Jacobian matrix is

$$
f^{\prime}(x, y, z)=\left[\begin{array}{lll}
\frac{\partial f_{1}}{\partial x} & \frac{\partial f_{1}}{\partial y} & \frac{\partial f_{1}}{\partial z} \\
\frac{\partial f_{2}}{\partial x} & \frac{\partial f_{2}}{\partial y} & \frac{\partial f_{2}}{\partial z} \\
\frac{\partial f_{3}}{\partial x} & \frac{\partial f_{3}}{\partial y} & \frac{\partial f_{3}}{\partial z}
\end{array}\right]=\left[\begin{array}{l}
A_{11} A_{12} A_{13} \\
A_{21} A_{22} A_{23} \\
A_{31} A_{32} A_{33}
\end{array}\right]
$$

When the Jacobian matrix is non-singular, the target position can be expressed as

$$
\left[\begin{array}{l}
x^{k+1} \\
y^{k+1} \\
z^{k+1}
\end{array}\right]=\left[\begin{array}{l}
x^{k} \\
y^{k} \\
z^{k}
\end{array}\right]-f_{i}(x, y, z)^{-1}\left[\begin{array}{l}
f_{1}\left(x^{k}, y^{k}, z^{k}\right) \\
f_{2}\left(x^{k}, y^{k}, z^{k}\right) \\
f_{3}\left(x^{k}, y^{k}, z^{k}\right)
\end{array}\right]
$$
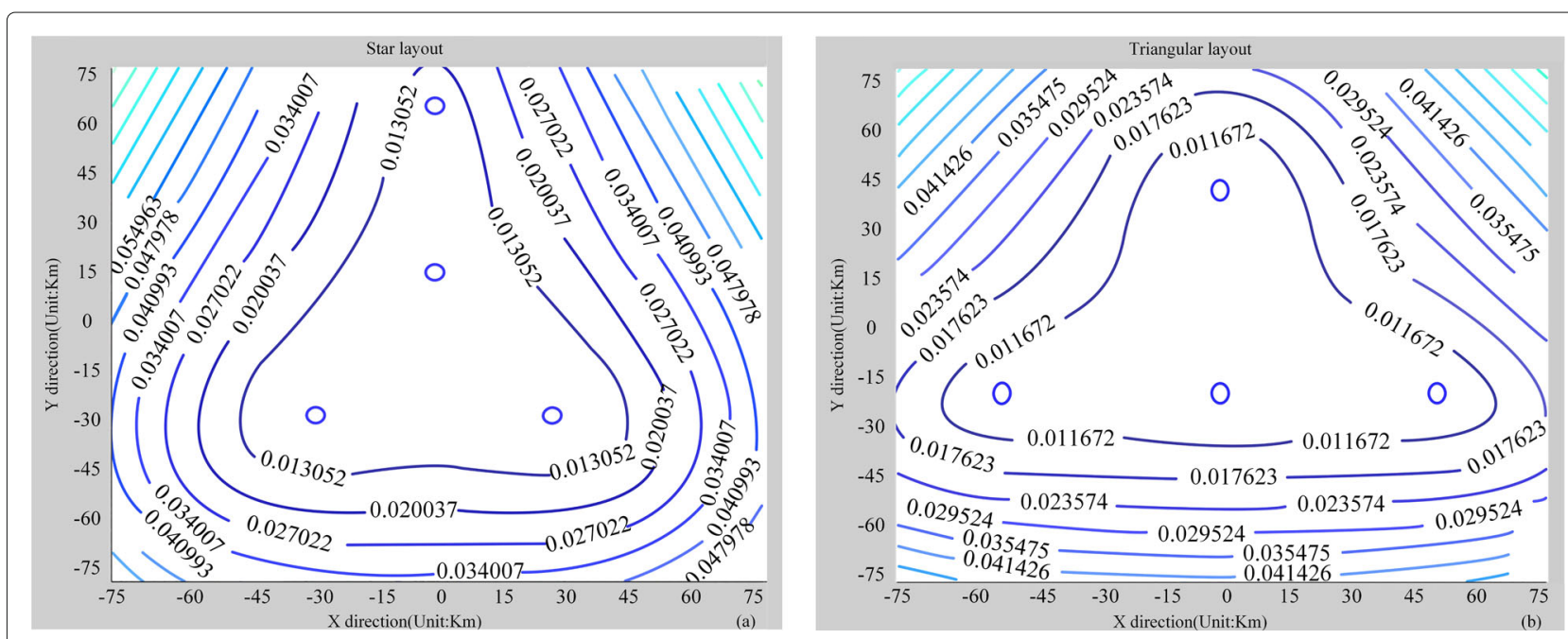

Fig. 2 Accuracy comparison. Accuracy comparison between star layout (a) and triangular layout (b) 

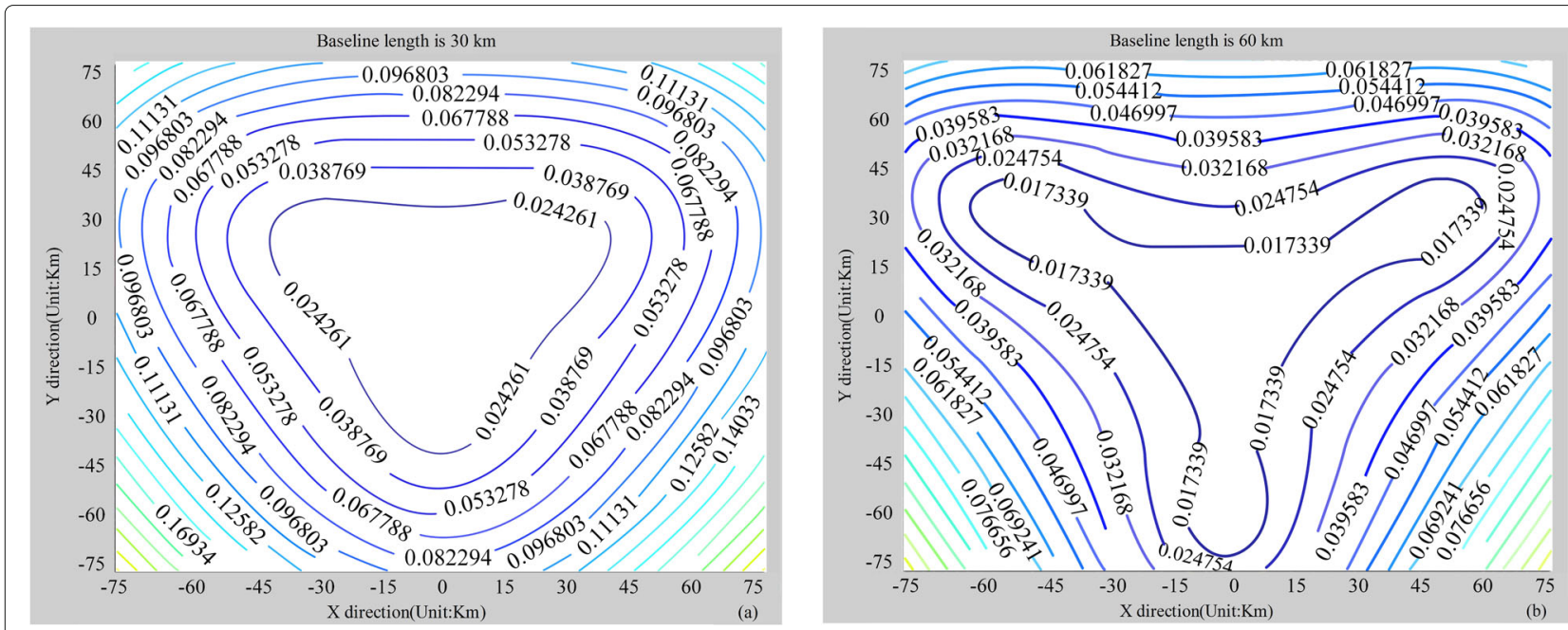

Fig. 3 Influence of baseline length to positioning accuracy

\section{Numerical analysis of the proposed algorithm}

When we use passive positioning system to locate a target in the air, the layout of its base stations has a considerable impact on the positioning accuracy [21]. As reported in the literature (e.g., [22]), when the target's flight direction or trend remains uncertain, the star-shaped layout will yield a more accurate position. Next, we simulate the factors that influence the positioning accuracy under star layout. We set the time error of base station is $10 \mathrm{~ns}$ and the site measurement error is $6 \mathrm{~m}$, the correlation coefficient of the time error and the flattened measurement error is 0.1 . Firstly, we verify the impact of the baseline length to positioning accuracy. We set the target height is $8 \mathrm{~km}$ and set the baseline lengths as $30 \mathrm{~km}$ and $60 \mathrm{~km}$, respectively. Figure 3 shows that the smaller baseline length, the lower positioning accuracy. However, in a real environment, the baseline length cannot be too long because of geographical environment and communication between base stations. Then, we verify the impact of targets at different heights on positioning accuracy. We set the baseline length as $30 \mathrm{~km}$ and set the target heights as $10 \mathrm{~km}$ and $20 \mathrm{~km}$, respectively. Figure 4 shows that the lower height, the lower positioning accuracy. Under the star layout, the target height is set to $20 \mathrm{~km}$, and the positioning accuracy curve is distributed with an ellipsoidal isoline with the main station as the center point.

Finally, we verify the influence of the height of the base station on the positioning accuracy. We set the distance between the base stations as $30 \mathrm{~km}$ and set the height of the base stations as $0.1 \mathrm{~km}$ and $0.3 \mathrm{~km}$, respectively.
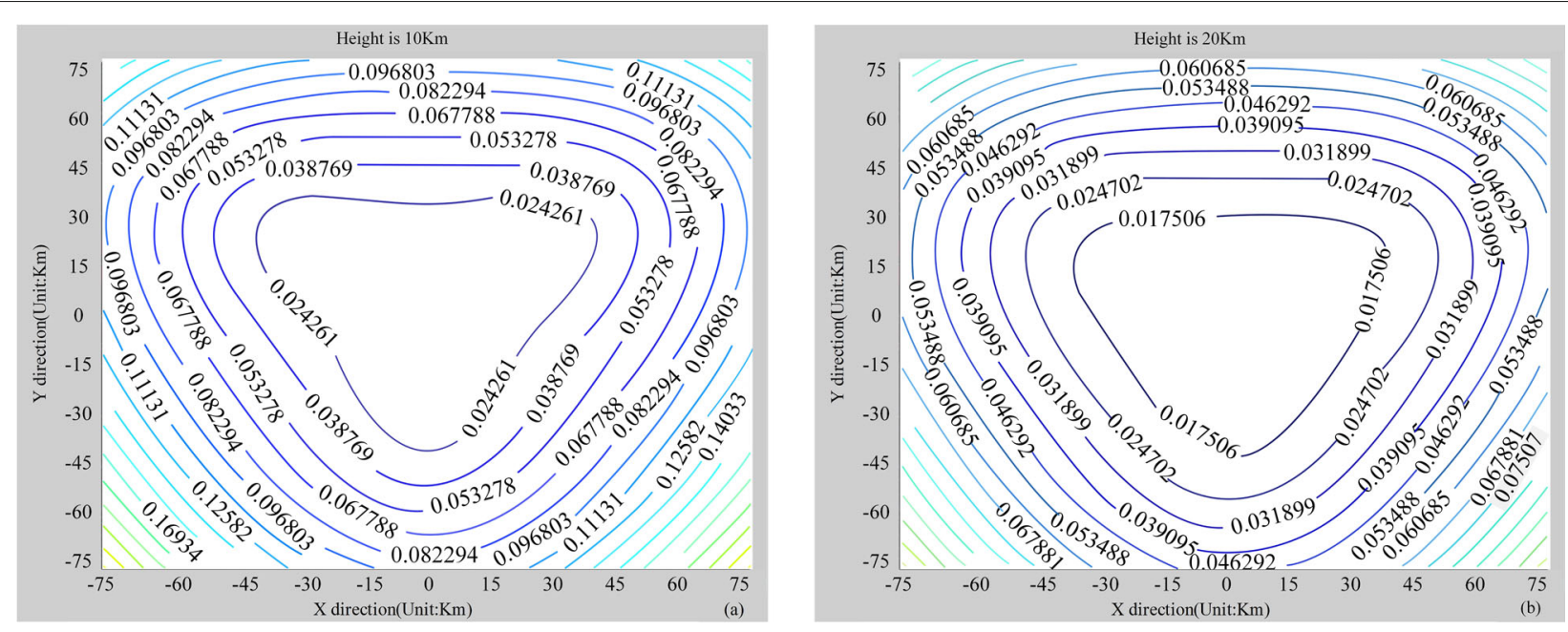

Fig. 4 Influence of different target height on positioning accuracy 

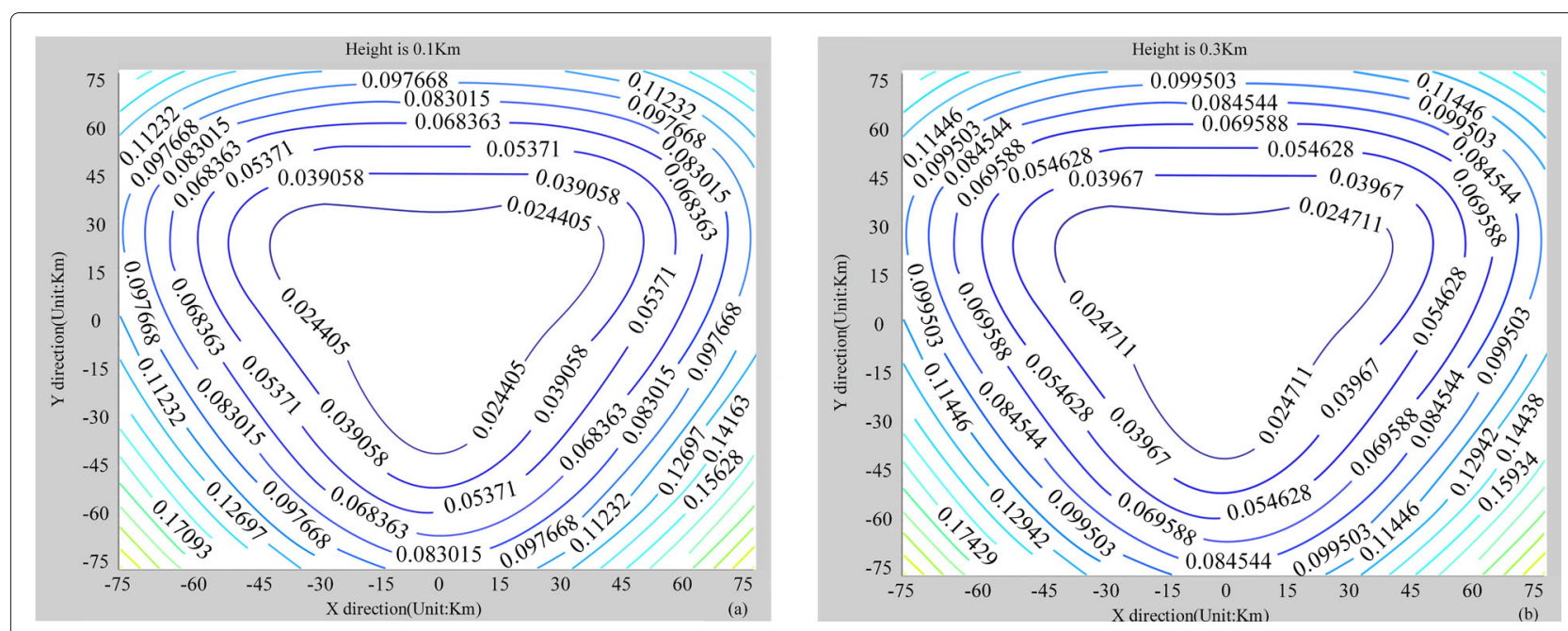

Fig. 5 Influence of the height of the base station on positioning accuracy

According to Fig. 5, when the height between stations decreases, the positioning accuracy will increase. Compared with Figs. 3 and 4, it shows that changing the baseline length and the height of the target has a greater impact on positioning accuracy than changing the height of the base station. According to the simulation, it can be observed that the farther the target is from the main station in the measurement range, the lower the positioning accuracy is. The positioning accuracy curve can be approximated as an equivalent circle centered on the master station after several simulation shows. So it meets the basic requirements of positioning accuracy. Therefore, the star layout is the best choice after taking all the abovementioned elements into consideration.

When simulating the target positioning system, we set the coordinates of main stations $O, A, B$, and $C$ as $(0$,
$0,0),(26,15,0),(-26,15,0)$, and $(0,30,0)$, respectively. The unit is kilometer, the time measurement error is set to $10 \mathrm{~ns}$, the angle between the base stations is $\varphi_{1}=\varphi_{2}=120^{\circ}$, and the baseline length is $L=$ $L_{1}=L_{2}=L_{3}=30 \mathrm{~km}$. As shown in Fig. 6, the target keeps uniform rectilinear motion at altitude $5 \mathrm{~km}$ above the $x-y$ plane ( $M N$ is the motion path). We average over one hundred execution of the algorithms with different initialization and analyze the root mean square error of the target path to test the performance of the proposed hybrid algorithm. The analysis result is shown in Fig. 7. The minimum error is $5 \mathrm{~m}$ when the sampling sequence is between 40 and 70 , which shows that the algorithm can effectively improve the three-dimensional positioning accuracy of the four-station TDOA positioning system. And the maximum positioning error is less

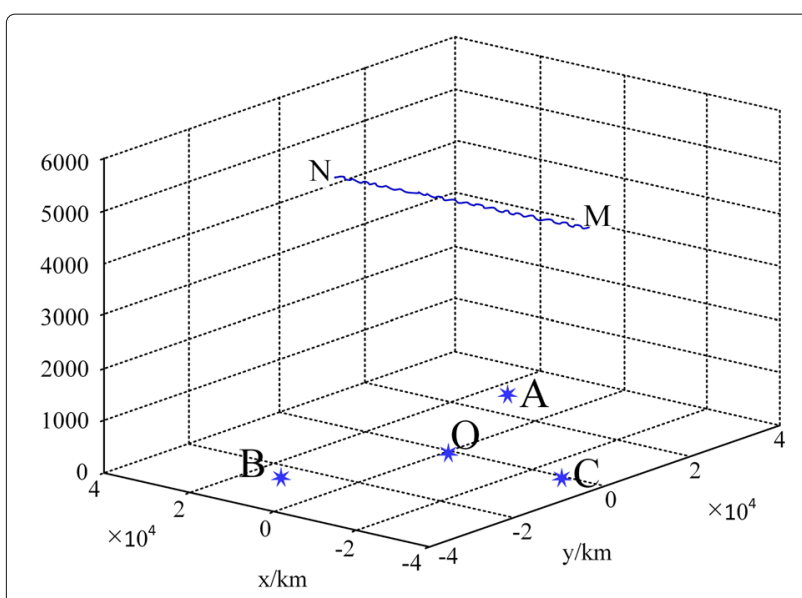

Fig. 6 Motion path of the target

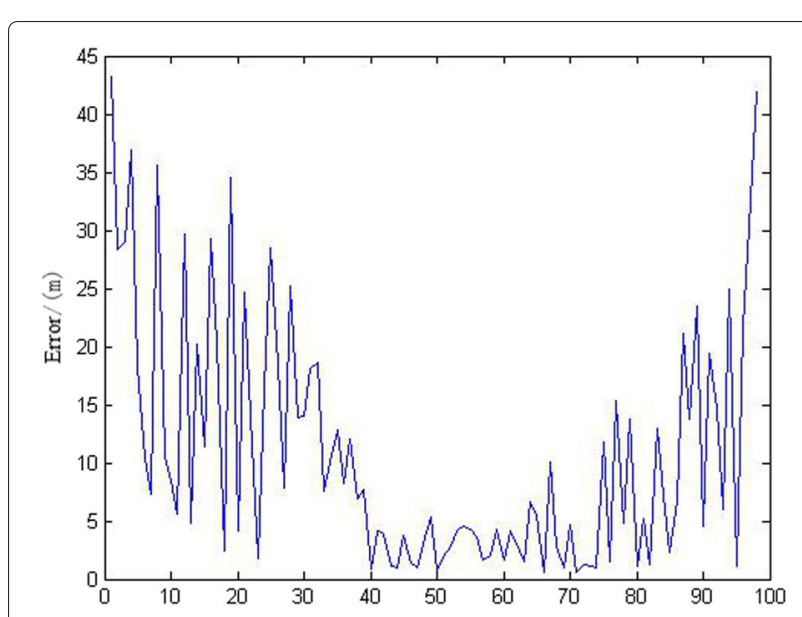

Fig. 7 Analysis of root mean square error simulation 
Table 1 Accuracy comparison between the two location algorithms

\begin{tabular}{|c|c|c|c|c|c|c|}
\hline \multirow{2}{*}{ Target's exact coordinates } & \multicolumn{3}{|c|}{ The least square algorithm } & \multicolumn{3}{|c|}{ Conversion algorithm based on spherical coordinates } \\
\hline & $X$-axis error & $Y$-axis error & Z-axis error & $X$-axis error & $Y$-axis error & Z-axis error \\
\hline$[-116,-62,8]$ & -0.1857 & -0.1016 & -0.1424 & -0.1009 & -0.0470 & 0.0326 \\
\hline$[50,28,8]$ & 0.0137 & 0.0073 & 0.0799 & 0.0153 & 0.0110 & 0.0195 \\
\hline$[200,180,30]$ & 0.5151 & 0.4794 & 0.3249 & 0.3793 & 0.3567 & 0.0043 \\
\hline$[100,181,10]$ & 0.2055 & 0.4137 & 0.2259 & 0.1854 & 0.3341 & -0.1986 \\
\hline$[-150,200,5]$ & 0.2663 & -0.3826 & -0.4472 & -0.1494 & 0.1948 & 0.4513 \\
\hline$[100,120,100]$ & 0.1280 & 0.1665 & 0.1706 & 0.0993 & 0.1232 & 0.0962 \\
\hline$[160,140,50]$ & 0.3118 & 0.2836 & 0.1989 & 0.2291 & 0.2117 & 0.0584 \\
\hline$[50,50,100]$ & 0.0097 & 0.0116 & 0.0438 & 0.0242 & 0.0254 & 0.0513 \\
\hline$[30,30,1]$ & 0.0051 & 0.0070 & 0.4102 & 0.0085 & 0.0103 & 0.0253 \\
\hline$[50,50,10]$ & 0.0192 & 0.0230 & 0.0555 & 0.0262 & 0.0298 & 0.0028 \\
\hline$[60,40,3]$ & 0.0273 & 0.0189 & 0.2414 & 0.0248 & 0.0201 & 0.0370 \\
\hline
\end{tabular}

than $45 \mathrm{~m}$, which is acceptable in most engineering applications.

To further evaluate the performance of this algorithm, we compare it against the least square method through simulation in different areas by measuring errors and accuracy. Results in Table 1 show that the proposed hybrid algorithm achieves the same location accuracy of TDOA algorithm based on the least squares method. (i) The least square algorithm can obtain the coordinates of the target by operating weighted least square method twice. It features high accuracy and low calculation complexity when the noise obeys the Gauss distribution. The least squares method requires at least five base stations and is subject to time difference measurement error. (ii) The $z$-axis location accuracy of the proposed hybrid algorithm is always higher than that of the least squares algorithm. (iii) When the distance between the target and the base stations becomes larger, the location accuracy of our algorithm will be better than that of the least square algorithm.

\section{Conclusion}

In this paper, we propose a joint algorithm that can achieve high-precision positioning of TDOA and fast convergence of Newton's method. After the algorithm is integrated with the spherical coordinate transformation method, it can improve the fuzzy positioning and the inconsistent positioning equations, so that it can be used for low-altitude target positioning. Compared with least square method and Newton's method, the positioning accuracy has been significantly improved, especially the $z$-axis, and the proposed method is better than the others. The results further indicating the algorithm can effectively improve the three-dimensional positioning accuracy of the four-station TDOA positioning system. The positioning results are more precise even if the long distance between the target and the base stations, and the positioning accuracy achieved within $45 \mathrm{~m}$, which provides a suitable solution for engineering applications. Different layouts of the base stations will affect the positioning accuracy, and the positioning effect reaches the best effect when the system is a star-shaped station. For more complex scenarios in the future, we can further analyze the influence factors of the positioning algorithm. The positioning accuracy is not only related to the spatial position of the target and each base station, but also affected by factors such as the location of the reconnaissance station, the geographical location of the positioning base station, and the time difference measurement error and base station position measurement error. With the involvement of a large number of IoT devices in the future, there will be higher requirements for the real-time and reliability of target positioning accuracy, which is also an important direction for future research.

\section{Abbreviations}

TDOA: Time difference of arrival

\section{Acknowledgements}

Not applicable.

\section{Authors' contributions}

QJS, SHN, QN, WC, and SC carried out all research steps including finding the problem,analyzing the related works, and finding, implementing, and simulating the proposed method as well as writing the manuscript. AR has contributed in analyzing the data and editing the paper. All authors read and approved the final manuscript.

\section{Authors' information}

Junsuo QU received his B.S. degree in the telecommunication engineering from Chongqing Institute of Posts and Telecommunications, Chongqing, China, in 1991, and M.S. degree in the communication and information system from Xidian University, Xi'an, China, in 1998. He is a faculty member of the School of Communication and Information Engineering from Xi'an University of Posts and Telecommunications as a Full Professor. He is the person in charge in Xi'an Key Laboratory of Advanced Control and Intelligent Process, Xi'an, China, and a Member of the Chinese Association of Communication. Haonan SHI received a bachelor's degree in communication engineering from Mingde College of Northwestern Polytechnical University in China in 2018. Now, he is studing for a master's degree in Xi'an University of Posts and 
Telecommunications. He is 23 years old, and his main research topic is the technology and application of the Internet of Things.

Ning QIAO received a bachelor's degree in communication engineering from Xi'an University of Posts and Telecommunications in China in 2016. Now, he is studying for a master's degree in Xi'an University of Posts and Telecommunications. He is 25 years old, and his main research topic is deep learning and big data analysis.

Chen WU received a bachelor's degree in communication engineering from Xi'an University of Posts and Telecommunications in China in 2016. Now, he is studying for a master's degree in Xi'an University of Posts and Telecommunications. He is 25 years old, and his main research topic is the technology and application of the Internet of Things.

CHANG SU received the B.S. degree in Electronics Science and Technology from Ningxia Normal University, Guyuan, China, in 2017. Now, he is studying for a master's degree in Xi'an University of Posts and Telecommunications. He is 25 years old and his main research topic is the technology and application of the Internet of Things.

Abolfazl Razi is an Assistant Professor of Electrical Engineering in the School of Informatics, Computing and Cyber Systems (SICCS) and the director of Wireless Networking and Smart Health (WiNeSH) research laboratory at Northern Arizona University. He received the B.Sc. degree in electrical engineering from Sharif University, the M.Sc. degree from Tehran Polytechnic, and the Ph.D. degree in electrical engineering from the University of Maine. Prior to joining NAU, he held post-doctoral position with the Electrical and Computer Engineering Department at Duke University, where he developed novel information-theoretic methods for dictionary learning, compressive sensing, and inverse problems. He also held a postdoctoral associate position in Case Western Reserve University, where he developed computational methods based on Bayesian inference for integrative analysis of cancer omics data. In addition to his academic service, Dr. Razi served about 7 years in wireless industry holding several positions including project manager of value added services, RandD researcher, network optimization and integration engineer, and smart card design and test engineer.

\section{Funding}

This work is partially supported by the National Natural Science Foundation of China under Grant 51875457, International Cooperation and Exchange Program of Shaanxi Province under Grant 2018KW-026, Natural Science Foundation of Shaanxi Province under Grant 2019JM-606, Natural Science Foundation of Shaanxi Province under Grant 2018JM6120, and Xi'an Science and Technology Projects under Grant 201805040YD18CG24(6).

\section{Availability of data and materials}

Data sharing is not applicable to this article as no datasets were generated or analyzed during the current study.

\section{Competing interests}

The authors declare that they have no competing interests.

\section{Author details}

${ }^{1}$ School of Automation, Xi'an University of Posts and Telecommunications, 710121 Xi'an, China. ${ }^{2}$ School of Communication Engineering, Xi'an University of Posts and Telecommunications, 710121 Xi'an, China. ${ }^{3}$ School of Informatics, Computing and Cyber Systems, Northern Arizona University, 86011 Flagstaff, USA.

Received: 16 September 2019 Accepted: 6 March 2020

Published online: 08 April 2020

\section{References}

1. Y. Zou, H. Liu, X. Wei, Q. Wan, Semidefinite programming methods for alleviating sensor position error in TDOA localization. IEEE Access. PP, 1-1 (2017)

2. P. Wu, X. Li, L. Zhang, Y. Bo, Passive location using TDOA measurements from compass satellite illuminators. Asian J. Control. 17, 722-728 (2015)

3. G. Q. Zhou, L. J. Yang, Z. Liu, N. I. Lei-Lei, Influence of base station deployment on location precision and fuzzy area distribution based on TDOA location. J. Nav. Univ. Eng. 25, 96-101 (2017)

4. Y. Jiang, M. Liu, C. Tao, L. Gao, TDOA passive location based on Cuckoo search algorithm. J. Shanghai Jiaotong Univ. (Sci.) 23, 368-375 (2018)
5. Z. Y. Wang, N. Xia, W. L. Yang, Research and design of high precision shortwave positioning system based on TDOA. Comput. Meas. Control. 9 , 3144-3147 (2015)

6. Y. Tuo, Y. Wang, S. Wang, Reliability-based robust online constructive fuzzy positioning control of a Turret-Moored floating production storage and offloading vessel. IEEE Access. 6, 36019-36030 (2018)

7. L. L. Yang, X. W. Sun, Precision analysis of airborne passive location of multi-stations. Acad. China Acad. Electron. Inform. Technol. 9, 348-352 (2014)

8. J. Wang, X. J. Cheng, J. Q. Liu, Y. J. Wen, A Enhanced algorithm based on RSSI and quasi Newton method for the node localization in wireless sensor networks. Comput. Knowl. Technol. 12, 222-225 (2016)

9. G. Q. Zhou, L. J. YANG, Z. Liu, Analysis on the influence of base station layout on the fuzzy region distribution and positioning accuracy based on TDOA positioning. J. Nav. Univ. Eng. 29, 96-101 (2017)

10. C. Dai, L. Song, D. Chao, Approach for 3D localization based on RSSI of 4 nodes. J. Comput. Meas. Control. 24, 229-232 (2016)

11. J. S. QU, X. N. HOU, J. R. ZHANG, 3D localization algorithm based on 4-station time difference of arrival and Newton iteration. J. Xi'an Univ. Posts Telecommun. 20, 36-40 (2015)

12. G. Yuan, J. Chen, Solution on ambiguity of time difference location system of three stations. Acad. Res. Electron. Chin. Acad. Sci. 9, 89-92 (2014)

13. S. Sun, Z. Wang, Z. Wang, in International Conference on Computer Engineering, Information Science and Application Technology. Study on optimal station distribution based on TDOA measurements, (2016). https://doi.org/10.2991/iccia-16.2016.53

14. Y. F. Hang, R. G. Dou, L. I. Hao, J. Q. Dai, S. Y. Pan, Coordinate system conversion of GPS control network on surface movement observation station based on the robust estimation. Beijing Surv. Mapp. 6, 77-82 (2013)

15. H. J. Zhu, Y. Zhang, E. Mei, Y. U. Hua-Gang, Constrained weighted least-squares algorithm for moving source location using TDOA and FDOA measurements. Telecommun. Eng. 53, 395-401 (2013)

16. W. Yue, K. C. Ho, Unified near-field and far-field localization for AOA and hybrid AOA-TDOA positionings. IEEE Trans. Wirel. Commun. 17, 1242-1254 (2018)

17. Z. Yi, Y. Wu, J. Yan, H. Wang, 3D inversion of full gravity gradient tensor data in spherical coordinate system using local north-oriented frame. Earth Planets Space. 70, 58-58 (2018)

18. J. F. Yang, P. Z. Zhang, Research on seismic source localization method based on DTOA/DOA and Newton iterated algorithm. J. Seismol. Res. 36, 324-329 (2013)

19. F. B. Gunay, T. Cavdar, in Signal Processing and Communications Applications Conference. Mobile fleet localization model via RSSI, TOA and TDOA in wireless sensor networks, (2014). https://doi.org/10.1109/siu. 2014.6830508

20. L. I. Jinzhou, F. C. Guo, W. L. Jiang, Source localization and calibration using TDOA and FDOA measurements in the presence of sensor location uncertainty. Sci. China Inf. Sci. 57, 1-12 (2014)

21. Z. Tong, C. Zhang, Z. Sheng, Z. Wang, in Consumer Communications and Networking Conference. On the performance of wireless source-location using TDOA measurements under poor geometry, (2016). https://doi.org/ 10.1109/ccnc.2016.7444918

22. L. Xi, F. C. Guo, Y. Le, Z. Min, Improved solution for geolocating a known altitude source using TDOA and FDOA under random sensor location errors. Electron. Lett. 54, 597-599 (2018)

\section{Publisher's Note}

Springer Nature remains neutral with regard to jurisdictional claims in published maps and institutional affiliations. 